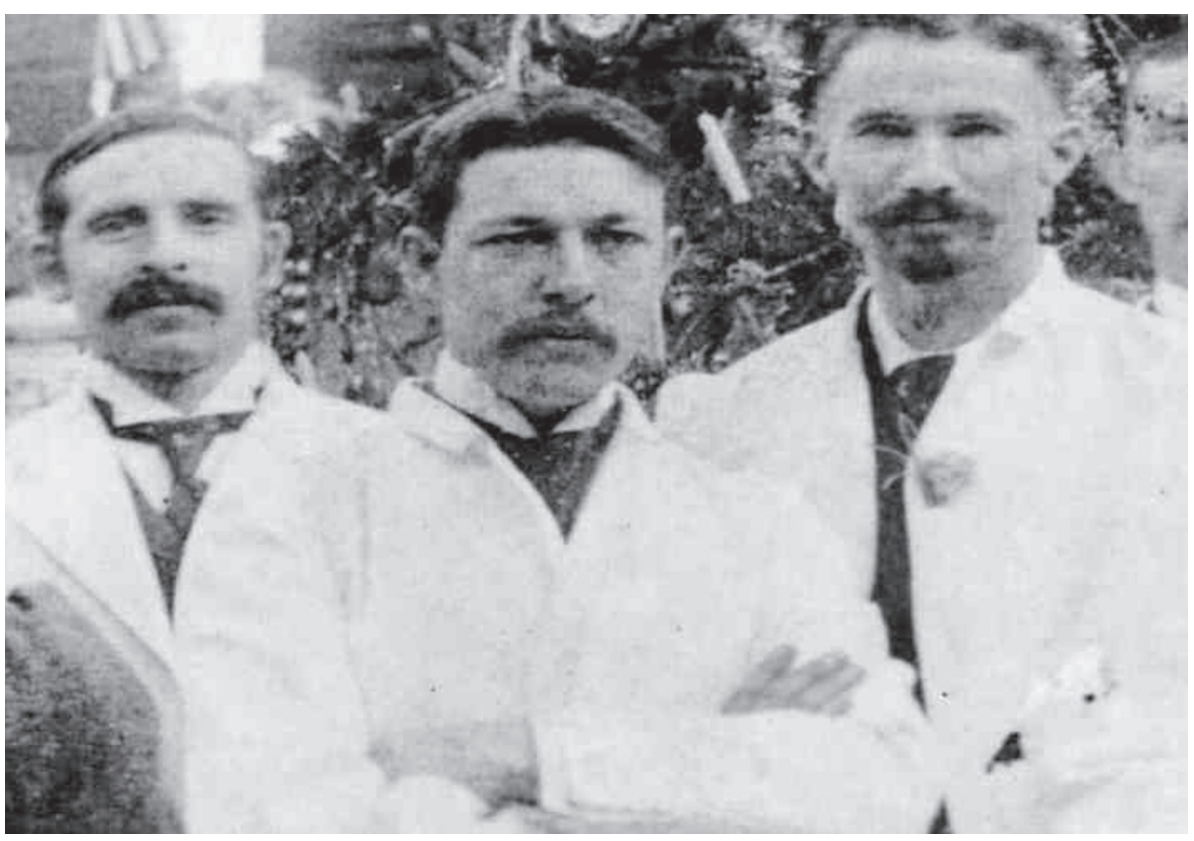

William Coley (centre) was the first to practise cancer immunotherapy a century ago.

BACTERIOLOGY

\title{
A caring culture
}

\section{William Coley found a way to prompt the immune system to fight cancer over a century ago. After years of neglect, scientists are now seeking to replicate his success.}

\section{BY SARAH DEWEERDT}

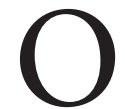
ne day in October 2005, while working on what he hoped would become a widely effective cancer therapy, MacAdam went down to the Yale University archives to pore over hundred-year-old patient records, taking notes by hand. Back in the lab, his colleagues had tracked down an old-fashioned strain of bacteria, isolated from a patient who died of scarlet fever in 1924, and were experimenting with culture techniques found in dusty bacteriology textbooks - even growing the bugs in ground beef, a common approach in the nineteenth century.

MacAdam, chief executive of MBVax Bioscience, based in Vancouver, Canada, was attempting to replicate the success of a cancer vaccine developed at the end of the nineteenth century by a young doctor named William Coley.

Coley turned to bacteria to save his patients' lives. In 1890, wracked with regret over the death of his first patient from a soft-tissue cancer called a sarcoma, he searched the medical literature for anything that might have saved her. He read about another sarcoma patient whose tumour had mysteriously disappeared after a bacterial skin infection. Coley tracked the man down and found that he remained cancer free seven years after the infection.

The case was not unique. Coley soon found documented examples going back hundreds of years in which others had experienced such 'spontaneous regression' of cancer after infection. Reasoning that the infections could have prompted these patients' immune systems to fight their tumours, he turned to the bacteria that cured the man he had met. He deliberately infected one of his own sarcoma patients with Streptococcus pyogenes, and within weeks his patient made a dramatic recovery.

Coley continued to administer his therapeutic cancer vaccine to patients over the next four decades, tinkering with it along the way. He began using heat-killed bacteria to make the treatment safer, and added a second species to improve its effectiveness. He treated hundreds of people, curing more than a quarter of his sarcoma patients plus some with other types of cancer.

Even by today's standards, his results are remarkable. In 1999, researchers compared 128 of Coley's cases with 1,675 matched controls treated with modern cancer therapies, and found that his patients survived a median of 8.9 years, compared with
7.0 years for contemporary patients. Half of Coley's sarcoma patients lived for ten years, compared with $38 \%$ of those treated with modern therapy. Coley also improved the tenyear survival rates for patients with kidney and ovarian cancers. "What Coley did for his [sarcoma] patients back then was better than what we're doing for these same patients today," says Charlie Starnes, a researcher at Amgen, a biotechnology company based in Thousand Oaks, California, who has studied the history and mechanisms of Coley's therapy.

But after more than a century, the field of immunotherapy that Coley launched still hasn't come to fruition. Radiation and chemotherapy became established therapies in the mid-1900s, pushing Coley's vaccine out of favour. Chemotherapy and radiation were comparatively easy to standardize, while Coley's approach required careful calibration for each patient and didn't seem to work as well for other cancers as it did for sarcoma. Scientists didn't understand the underlying mechanisms, and some doctors couldn't replicate the results at all.

That may finally be changing, thanks to a better understanding of how the immune system functions, of the molecular mechanisms underlying infection and cancer regression, and of previously ignored details of Coley's work.

\section{RECONSTRUCTING SUCCESS}

Over the course of Coley's career he worked with many different bacteriologists who made more than 20 versions of the vaccine; some were more effective than others. "The vaccine that was made by a bacteriologist named Martha Tracy was clearly the most successful version," says Stephen Hoption Cann, chief medical officer at MBVax.

So the MBVax team set about trying to replicate Tracy's vaccine using modern laboratory techniques - and sterile culture media rather than ground beef. The reconstructed vaccine contains killed S. pyogenes, a spherical bacterium that commonly infects the throat and skin, and Serratia marcescens, a bright red, rod-shaped bacterium that contains an immune-stimulating pigment known as prodigiosin.

MBVax hasn't yet conducted controlled trials of its vaccine. But from 2007 to 2012, the company gave it to about 70 people with late-stage cancers, including melanoma, lymphoma and malignancies in the breast, prostate and ovaries. Tumours shrank in about $70 \%$ of the patients, and $20 \%$ went into complete remission, according to MBVax.

Several other groups are researching similar mixed-bacteria vaccines. In 2012, researchers in Germany tested a combination of heat-killed S. pyogenes and S. marcescens bacteria in a phase I safety trial in 12 cancer patients, and found that the vaccine increased levels of cytokines that enhance immune response. And even though it wasn't the aim of their study, they also documented tumour 
¿ regression in one participant.

Not all patients respond, however, and MBVax wants to know why. But before it can do a clinical trial to answer those questions, the company must build a multimilliondollar production facility that meets US and European standards for manufacturing pharmaceuticals. For now, MBVax has stopped vaccine production, but Hoption Cann says the company is seeking funding for the facility and hopes to begin development within two years.

Such difficulties are typical of the regulatory hurdles that any revival of Coley's therapy is likely to face. "In drug development, it's very difficult to get approval for a bacterial extract," says Uwe Hobohm, a biologist and bioinformaticist at the University of Applied Sciences in Giessen, Germany, who champions Coley's work. Instead, regulatory agencies prefer to see therapies based on individual molecules with well-defined mechanisms of action, rather than bacteria-based products that can include multiple active and inactive molecules and a variety of mechanisms.

\section{MOLECULES AND MECHANISMS}

Hobohm says that a more feasible approach might be to determine the mechanisms by which mixed-bacterial vaccines work, and to develop therapies based on specific molecules produced by bacteria but purified and standardized to make them acceptable to the US Food and Drug Administration (FDA). He believes Coley's success can be traced to a class of molecules known as pattern recognition receptor (PRR) ligands, which lend themselves well to this kind of regulated production.

Pathogens produce a variety of PRRs, including lipopolysaccharides, certain proteins and DNA. These molecules activate dendritic cells - a type of immune cell that recognizes pathogens and coordinates the initial stages of the immune response. In the past, many researchers thought the immune system did not attack cancer because it didn't recognize malignant cells as foreign. But Hobohm thinks that is only half the story, as a dendritic cell must encounter PRR ligands before it can fully activate $\mathrm{T}$ cells, in turn enabling them to recognize and destroy cancer cells. But because cancer cells don't make PRR ligands, the dendritic cells can't generate a robust response. "Usually the immune reaction is there, but it's not strong enough," Hobohm

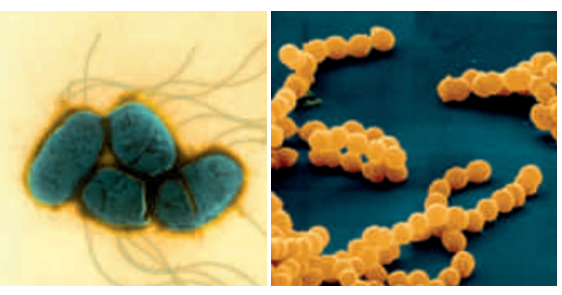

Serratia marcescens (left) and Streptococcus pyogenes infections can have anticancer effects.

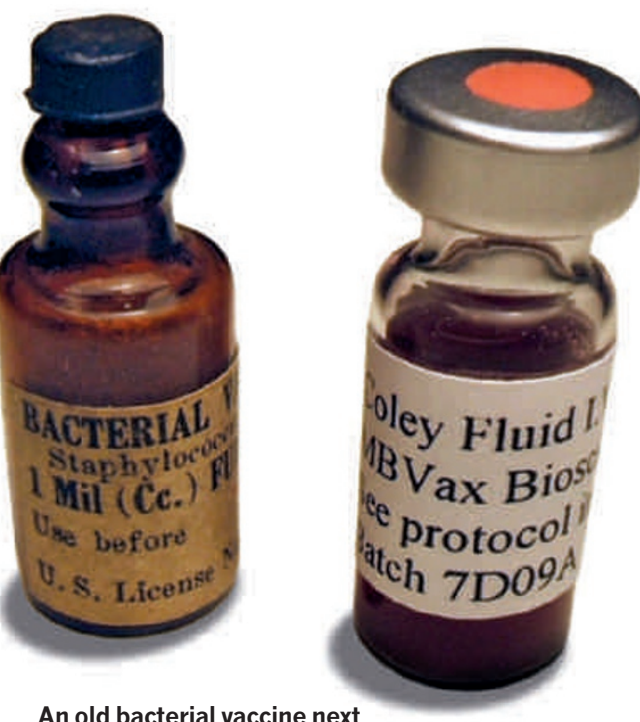

An old bacterial vaccine next to its contemporary version.

says. "We believe that the proper activation by dendritic cells is missing because PRR ligands are missing."

Hobohm tested this theory by administering commercially available PRR ligands, which are purified from bacteria, to mice with experimentally induced tumours. Other researchers had previously tested only single PRR ligands, or multiple PRR ligands in combination but with only a few doses. By contrast, Coley administered his vaccine at least once or twice a week for several weeks or even months. Following Coley's lead, Hobohm gave his animals injections of three PRR ligands ten times over the course of three weeks. The approach cured four out of the five mice in the study.

One of the molecules used in the study, mistletoe lectin, is widely used as an addon cancer therapy in Europe but was only recently shown to be a PRR ligand. Hobohm found that its structure is strikingly similar to that of a toxin produced by the bacterium Shigella dysenteriae, implying that, like the toxin, it probably triggers an immune response. Similarly, imiquimod, which is used to treat skin cancer, is thought to be a PRR ligand because of the way it interacts with immune cells. Hobohm believes that it might be possible to increase the efficacy of both therapies by combining them with other PRR ligands.

One barrier preventing further investigation of PRR therapy, however, is that the molecules often induce a fever - much like the bacteria from which they originate. And because fever usually indicates infection, it is classified as a negative side effect in drug development studies. Indeed, early versions of Coley's therapy, which included live bacteria, sometimes killed his patients. But those patients who had high fevers after receiving Coley's vaccine had better survival rates than those who experienced little or no fever.

Hoption Cann says unpublished data show that patients treated with the MBVax vaccine are more resistant to infection than other cancer patients, despite frequent treatment-induced fevers, which suggests that the fever is not harmful. Yet the prejudice against fever has made it hard for some researchers to get their studies funded, he adds.

\section{SITE INSIGHT}

Others doubt that fever is necessary, however. "Is temperature critical, or is it a bystander?" asks Simon Sutcliffe, chief medical officer of Qu Biologics in Vancouver, Canada. His company is taking a different Coley-inspired approach, and has seen promising results in patients with advanced cancer without causing a fever.

After reviewing Coley's data and spotting a pattern others hadn't detected before, $\mathrm{Hal}$ Gunn, the chief executive of Qu Biologics, began developing a type of therapy he calls sitespecific immunomodulators (SSIs). Different species of bacteria infect different parts of the body, and Gunn noticed that Coley's therapy was particularly effective against cancers located in tissues that are most vulnerable to infection with S. pyogenes - the original bacteria Coley's research had targeted. "It came to me that it might be stimulating an immune response that was site specific," Gunn recalls. $\mathrm{Qu}$ Biologics has since developed a suite of vaccines, each derived from a single, organspecific bacterial species: Escherichia coli for the bowel, Klebsiella pneumoniae for the lung, and so on.

Gunn and his colleagues speculate that their SSIs reset the immune system by mimicking the beneficial effects of infection at a tumour site. In particular, they believe that SSI molecules alter the activity of macrophages, a type of cell involved in the early stages of an immune response. The SSIs, they say, cause macrophages to shift from a response involved in tissue repair and cancer growth to a response that promotes the destruction of abnormal cells.

More than 250 patients with advanced cancer have been treated with SSIs from $\mathrm{Qu}$ Biologics. No randomized study of the therapy has been done, but an analysis by the non-profit Reliable Cancer Therapies found that SSI therapy increased median survival by 20 months among patients with advanced breast cancer, and by 12 months among those with a variety of late-stage cancers. Qu Biologics plans to begin clinical trials in late-stage lung-cancer patients in 2014.

Such studies fit into the larger vision held by advocates of Coley's work, who believe it is time to put historical research and inspiration to the test. "Clinical trials need to be carried out to show that this vaccine is beneficial for cancer patients," says Hoption Cann. "Until that time, interest in Coley's work comes and goes, but nothing stays around until you can demonstrate that." -

Sarah DeWeerdt is a freelance science writer based in Seattle, Washington. 